In press in Current Anthropology

\title{
Fission-Fusion Dynamics:
}

\section{New Research Frameworks}

${ }^{1}$ Filippo Aureli, Colleen M. Schaffner, Christophe Boesch, Simon K. Bearder, Josep Call, Colin A. Chapman, Richard Connor, Anthony Di Fiore, Robin I. M. Dunbar, S. Peter Henzi, Kay Holekamp, Amanda H. Korstjens, Robert Layton, Phyllis Lee, Julia Lehmann, Joseph H. Manson, Gabriel RamosFernández, Karen B. Strier, and Carel P. van Schaik

\footnotetext{
${ }^{1}$ Authors are listed in alphabetical order after the first three who organized the workshop that led to this collaborative effort.
}

Corresponding author:

Dr. Filippo Aureli

Research Centre in Evolutionary Anthropology and Palaeoecology

School of Biological and Earth Sciences, Liverpool John Moores University

James Parsons Building, Byrom Street, Liverpool L3 AF3, United Kingdom

Tel: +44-151-231 2488; Fax: +44-151-207 3224; Email: f.aureli@ljmu.ac.uk

\section{Acknowledgments}

The Wenner-Gren Foundation for Anthropological Research and Ethoikos financially supported the organization of the workshop where most of the ideas for the paper were discussed and developed. We are especially grateful to Roberto Cozzolino and his team for the smooth running of the workshop at the Convento dell’Osservanza, Radicondoli, Italy. We also thank 10 anonymous reviewers for 
comments. During the writing of this paper FA was supported by a HEFCE Promising Research Fellowship and RIMD by a British Academy Research Professorship.

FILIPPO AURELI is Professor and co-director of the Research Centre in Evolutionary Anthropology and Palaeoecology at Liverpool John Moores University (School of Biological and Earth Sciences, James Parsons Building, Byrom Street, Liverpool L3 AF3, United Kingdom [f.aureli@ljmu.ac.uk]). COLLEEN M. SCHAFFNER is Professor of Behavioral Biology in the Psychology Department, University of Chester. CHRISTOPHE BOESCH is Professor at the University of Leipzig and a director of the Max Planck Institute of Evolutionary Anthropology. SIMON K. BEARDER is Professor of Biological Anthropology, Oxford Brookes University. JOSEP CALL is co-director of the Wolfgang Köhler Primate Research Center, Max Planck Institute for Evolutionary Anthropology. COLIN A. CHAPMAN is Canada Research Chair and Professor of Anthropology and McGill School of Environment, McGill University. RICHARD CONNOR is Professor of Biology, University of Massachusetts-Dartmouth. ANTHONY DI FIORE is Associate Professor of Anthropology, Center for the Study of Human Origins, New York University. ROBIN I. M. DUNBAR is Professor of Evolutionary Anthropology, University of Oxford and co-director of the British Academy's Centenary Project "Lucy to Language”. S. PETER HENZI is Professor of Psychology, University of Lethbridge. KAY HOLEKAMP is Professor of Zoology, Michigan State University. AMANDA H. KORSTJENS is a Senior Lecturer in Biological Anthropology, Bournemouth University. ROBERT LAYTON is Professor of Anthropology, University of Durham. PHYLLIS LEE is Professor in Psychology, University of Stirling. JULIA LEHMANN is a Senior Lecturer in Biological Anthropology, University of Roehampton, JOSEPH H. MANSON is Professor of Anthropology, University of California at Los Angeles. GABRIEL RAMOS-FERNÁNDEZ is Professor of Biology, Instituto Politécnico Nacional, Mexico. KAREN B. STRIER is Professor of Anthropology, University of Wisconsin-Madison. CAREL P. VAN SCHAIK is Professor and director of the Anthropological Institute and Museum, 
University of Zürich. 


\begin{abstract}
Nineteen scientists from different disciplines collaborated in highlighting new methodological and theoretical aspects in the re-emerging study area of fission-fusion dynamics. The renewed interest in this area is due to the recognition that such dynamics may create unique challenges for social interaction and distinctive selective pressures acting on underlying communicative and cognitive abilities. In five sections, we outline new frameworks for integrating current knowledge on fissionfusion dynamics and suggest promising directions for future research using a broad comparative perspective. In the first section, we briefly review the diverse uses of the term "fission-fusion” and propose a fundamental re-thinking away from its current general use as a label for a particular modal type of social system (i.e., fission-fusion societies). Specifically, because the degree of spatial and temporal cohesion of group members varies both within and across taxa, we note that any social system can be described in terms of the extent to which it expresses fission-fusion dynamics, and we thus advocate a realignment of use of the term "fission-fusion" to reflect this perspective. The implications of this perspective are then discussed and expanded in three sections focusing on the socioecology, communication, and cognitive demands of fission-fusion dynamics. The last section explores the relevance of fission-fusion dynamics for human social evolution.
\end{abstract}




\section{Fission-Fusion Societies?}

${ }^{2}$ Filippo Aureli, Colleen M. Schaffner and Christophe Boesch

${ }^{2}$ These authors have principal responsibility for this section

The term "fission-fusion” was first introduced by Hans Kummer (1971) to describe the social system of a few taxa of nonhuman primates, such as chimpanzees, geladas, and hamadryas baboons, which change the size of their group by means of the fission and fusion of subunits (called parties or subgroups) according to both their activity and the availability and distribution of resources. Social systems characterized by such fission-fusion dynamics are considered rare among mammals, but they are typical of some primate (Smuts, Cheney, Seyfarth, Wrangham, and Struhsaker 1987) and other mammalian species (e.g., some bats: Kerth and König 1999; dolphins: Connor, Wells, Mann, and Read 2000; elephants: Moss and Lee in press; Wittemyer, Douglas-Hamilton, and Getz 2005; spotted hyenas: Holekamp, Cooper, Katona, Berry, Frank, and Smale 1997). Among primates most studies on fission-fusion dynamics have focused on chimpanzees (Boesch and Boesch-Achermann 2000; Mitani, Watts, and Muller 2002; Nishida and Hiraiwa-Hasegawa 1987) and spider monkeys (Chapman, Wrangham, and Chapman 1995; Symington 1990). Similar patterns also seem to occur in bonobos (Hohmann and Fruth 2002; Nishida and Hiraiwa-Hasegawa 1987; Stumpf 2007) and some populations of muriquis (Milton 1984). Fission-fusion dynamics are likewise typical of modern humans, including hunter-gatherers (Marlowe 2005), although it is not often explicitly recognized. The following quote captures this apparent anomaly: "Fission-fusion sociality seems so natural and necessary to humans including anthropologists - that it hardly demands explanation, if it is noticed at all” (Rodseth, Wrangham, Harrigan, and Smuts 1991:238). The sharing of such a flexible social nature with our closest living relatives suggests that fission-fusion dynamics was characteristic of the social system of the last common ancestor of chimpanzees, bonobos, and modern humans. 
Given the potential role of fission-fusion dynamics in human social evolution, we organized a symposium at the XXth Congress of the International Primatological Society and a post-congress workshop to explore from a broad comparative perspective whether fission-fusion dynamics create unique challenges for social interactions or distinct selective pressures for specific underlying communicative and cognitive abilities. In this section, we emphasize that the variation in fission-fusion dynamics goes beyond the modal types of societies that have traditionally been described as "fissionfusion". We therefore suggest a revision of how the term "fission-fusion” is used to reflect such variation, and we propose a new framework to stimulate the quantification of the relative degree of fission-fusion dynamics in different taxa in order to facilitate future comparative research.

\section{REDEFINING “FISSION-FUSION”}

Although the distinctions among modal types of "fission-fusion societies" (see electronic supplement A for a brief review) are useful to describe the degree of flexibility in the social system of certain species, many other systems characterized by fission-fusion dynamics do not fit within these modal types. In fact, there is pronounced variation in the degree of fission-fusion dynamics both across and within species (see electronic supplement B for examples), which poses a difficulty in using the modal terminology. Given this variation, we propose a fundamental re-thinking of that terminology. We suggest that the term "fission-fusion" be abandoned as a label for a particular modal type of social system (i.e., “fission-fusion societies”), and instead propose that the term "fission-fusion dynamics” be used to refer to the extent of variation in spatial cohesion and individual membership in a group over time. As a consequence, any animal society can be characterized by its degree of fission-fusion dynamics, which can vary from highly cohesive with stable group membership to highly fluid with either relatively stable or flexible subgroup membership.

This perspective on the use of the term “fission-fusion” fits well with Kappeler and van Schaik’s (2002) definition of social organization that explicitly incorporates the degree of spatiotemporal 
cohesion of a social system. In particular, this perspective emphasizes that simple dichotomous distinctions of social organizations as being cohesive versus flexible are neither realistic nor accurate (Strier 1989). Moreover, this perspective highlights that flexible spatiotemporal grouping patterns in primates and other vertebrates are more common - and more complex - than generally recognized (Kinzey and Cunningham 1994; Struhsaker and Leland 1979; Sussman and Garber 2007). The critical issue is that spatiotemporal variation in grouping patterns influences the opportunities for group members to interact with one another and, ultimately, the resulting social system.

Such a realignment of the use of the term "fission-fusion" requires the development of new conceptual frameworks for studying the implications of variation in fission-fusion dynamics across groups and species for socioecological, communicatory, and cognitive aspects. As a start, it is necessary to develop one or more basic heuristics for describing the degree of fission-fusion dynamics seen in a group or species and thus the relative opportunities that individuals have for close-range social interaction. Such a conceptual framework would, of course, be multi-dimensional. Here we propose a framework involving three dimensions that together capture potential temporal variation in spatial cohesion and membership in a given environment: (1) the temporal variation in spatial cohesion among group members, (2) the temporal variation in party size, and (3) the temporal variation in party composition (Figure 1; see electronic supplement C for detail on the three dimensions).

- Figure 1 about here -

The accumulation of data for the dimensions of the proposed framework, or other variants, would permit the relative placement of species and populations of the same species within a complex multidimensional fission-fusion space (e.g., Figure 1), which is an essential first step toward the systematic investigation of whether social systems characterized by different degrees of fission-fusion dynamics are quantitatively or qualitatively different from one another in terms of socioecological conditions, 
social interaction and cognitive abilities. However, as the relative positions of most species and populations within this multi-dimensional space have yet to be empirically determined, a simplified terminology is used throughout the following sections for ease of notation while making comparisons between social systems characterized by a higher degree of fission-fusion dynamics (hereafter "higher FF” groups or taxa) and those characterized by relatively higher temporal stability in group cohesion and membership (hereafter "lower FF" groups or taxa). We note that the terms "higher FF" and "lower FF” represent relative points within multi-dimensional fission-fusion space and therefore need to be interpreted within our relativistic approach of different degrees of fission-fusion dynamics (i.e., higher and lower in at least one of the three dimensions); that is, they do not reflect an actual dichotomy.

The three-dimensional framework proposed here is a useful first step, but additional complementary frameworks are needed, depending on which aspects of fission-fusion dynamics are being considered. The following sections propose several new frameworks that are relevant to exploring and understanding variation in fission-fusion dynamics in terms of socioecology, communication, and cognitive demands. The concluding section explores the implications of fissionfusion dynamics for human evolution.

\section{The Socioecology of Fission-Fusion Dynamics}

${ }^{3}$ Anthony Di Fiore, Colin A. Chapman, S. Peter Henzi, Phyllis Lee, Julia Lehmann and Gabriel RamosFernández

${ }^{3}$ These authors have principal responsibility for this section

Over the past 25 years, studies of primate behavior and ecology have contributed greatly to the development of socioecological theory, whose principal goal is to explain grouping patterns, range use, mating behavior, dispersal tendencies, and inter- and intra-sexual social relationships as adaptive responses to features of the ecological and social environment. Primate socioecology historically has 
taken a reductionist perspective, recognizing a discrete set of modal social systems and seeking to explain, for example, the "nepotistic" female social relationships of many cercopithecines as a consequence of the competitive regime engendered by food resource distribution. The discipline has come a long way using such a categorical framework, but this perspective downplays the fact that many aspects of social systems (e.g., the spatiotemporal variation in the association patterns of individuals) can vary dramatically, both among populations of a given taxon and within the same population over time. Primate socioecology is currently at an important juncture (Janson 2000). If primatologists are to continue to contribute to the development of socioecological theory, it is crucial for us to re-evaluate past assumptions about how ecological variables influence social systems and to expand our efforts to better understand the first principles that shape movement and grouping patterns. In the past, the study of higher FF taxa has offered a useful tool for exploring such issues, since their responses to ecological changes take place over very short time scales. Here, we return to the example of higher FF taxa and suggest a new framework that derives more explicit null models of association by using spatially-explicit agent-based simulations that begin from assumptions about the state of resources in the environment.

The modeling approach that we advocate will accomplish several goals. First, it will allow us to critically evaluate the validity of currently recognized modal types of primate social systems, particularly the so-called “fission-fusion societies” of chimpanzees and spider monkeys (see “Redefining Fission-Fusion” above). Second, the approach will let us explore, in silico, new metrics for describing patterns of socio-spatial association that might then be applied across taxa and be used for quantifying the fission-fusion space discussed above; using these metrics we can then begin to evaluate the importance of observed deviations from expected null models. Finally, through this approach we expect to be able to explore whether and how apparently similar social systems might arise for very different reasons and by different evolutionary routes (Lee 1994; see below). 


\section{ARE HIGHER FF TAXA “SPECIAL”?}

Because higher FF taxa are relatively uncommon among primates and other mammals, researchers tend to imagine that socioecological pressures they face may be somehow different in form or degree from those faced by lower FF taxa. Some higher FF groups (e.g., those falling into region C of Figure 1) have historically been incorporated into the general socioecological model as follows. First, the flexible association patterns of these higher FF groups are commonly interpreted as a solution by large-bodied primates - who face a relatively low risk of predation - for coping more efficiently with patchily distributed and temporally varying food sources (Dunbar 1988; Klein and Klein 1977; Milton 1984; Strier 1992; Symington 1988; van Schaik 1989; Wrangham 1977, 1980). Under these circumstances, females are suggested to spread out from one another when resources are scarce to reduce feeding competition, and possibly to aggregate and forage together when resources are plentiful. More recently, fission-fusion dynamics in these taxa have been viewed as affording flexible responses for optimal solutions to the usually contrasting pressures of avoiding predators and minimizing feeding competition when there is temporal or spatial fluctuation in predation pressure and food availability (Boesch and Boesch-Achermann 2000). For some higher FF taxa (e.g., chimpanzees, spider monkeys), males are or are presumed to be philopatric and cooperate with male relatives to defend access to the ranges of several females against males from neighboring groups (Ghiglieri 1984; Strier 1994; Wrangham 1979), which in turn is thought to force the dispersal of maturing females (Pusey 1979; Pusey and Packer 1987). Additional social and demographic factors (e.g., overall community size, presence of cycling females) have been shown to affect fission-fusion dynamics and party size (Lehmann and Boesch 2004). For other higher FF taxa (e.g., geladas and some baboons, which live in multilevel societies; region B of Figure 1) the risk posed by nocturnal predators is seen as the selective pressure favoring the aggregation of large numbers of animals at communal sleeping sites, while competition drives 
fissioning of these large groups into smaller, cohesive, foraging parties during the day (Dunbar 1988; Kummer 1968), and the risk of infanticide prompts the consistent association of particular males with particular females (Henzi and Barrett 2003).

There are a number of problems inherent in the notion that higher FF groups face "special” ecological pressures. First, past socioecological models of higher FF groups in primates offer only post hoc and incomplete explanations for observed patterns of association and kinds of social relationships. For example, the existence of a positive relationship between habitat-wide fruit availability and foraging party size in both spider monkeys and chimpanzees is taken as evidence that resource distribution constrains grouping patterns, and yet resource patch density and distribution explain less than half the variance in party size in these taxa (Chapman et al. 1995). In addition, long-term primate studies are increasingly revealing that considerable flexibility in grouping patterns and within-group social relationships may exist both between populations and within the same population of higher FF taxa over time (Strier 2003; see electronic supplement B). Thus, models of how ecological conditions shape even such basic aspects of social systems as group or party size are less than straightforward.

Moreover, as primatologists pay more attention to work on non-primate taxa, we are coming to appreciate that a wide variety of other vertebrates are also characterized by fission-fusion dynamics (see “Fission-Fusion Societies?” above and electronic supplement B). The extent to which socioecological models developed from primate studies can be applied across taxa is unknown, but it is very unlikely that any one set of similar ecological conditions determines superficially similar fissionfusion dynamics in these disparate taxa. Having identified a number of limitations to current socioecological models (see electronic supplement D) we outline a research framework that may help us to address these limitations. 


\section{A WAY FORWARD? DERIVING AN INCLUSIVE SPATIAL NULL MODEL}

Our proposition is to develop a new set of null models of association and sociality that begin from first principles to simultaneously explore links between ecological, social, and demographic variables on the one hand and the socio-spatial relationships on the other. Given that there is a great complexity in the environments in which grouping takes place, producing null expectations of grouping patterns should take as a starting point a spatially-explicit description of the environment inhabited by a species. Spatial analysis is a mature field that offers many tools useful to socioecologists for describing how resources (e.g., food, watering holes, sleeping sites) or predation risk vary in space and time (Dale, Dixon, Fortin, Legendre, Myers, and Rosenberg 2002; Fortin, Dale, and van Hoef 2002). From this description of the environmental complexity faced by animals, null models can be produced that predict what grouping patterns may arise simply as a consequence of the environment. One possible null model might be summarized as follows:

Within a primate social group, patterns of association across time are a consequence solely of individual decisions that maximize access to resources (i.e., they are not influenced by social factors, such as individuals' relative dominance rank or their relatedness to other group members). The temporal variation in spatial cohesion and membership characteristic of higher FF groups emerges in response to a specific subset of all possible patterns of resource dispersion.

Subsequently, deviation from what is predicted from these first principles can be analyzed to understand the relevant factors that, in addition to environmental variation, influence grouping patterns and social relationships. Some of these deviations will not lead too far (e.g., sleeping sites could physically constrain spatial dispersion), while others could be fascinating (e.g., quantitative 
descriptions of how alternative social strategies, such as infanticide avoidance, might influence grouping).

One way to develop null models of movement and grouping patterns against which empirical data can be compared is through the use of spatially-explicit agent-based simulations. Throughout the history of primate socioecological research, these kinds of models have been used by various researchers (Boyer, Miramontes, Ramos-Fernández, Mateos, Cocho, and Larralde 2004; Boyer, Ramos-Fernández, Miramontes, Mateos, Cocho, Larralde, Ramos, and Rojas 2006; Ramos-Fernández, Boyer, and Gómez 2006; Rodman and Di Fiore 1993; te Boekhorst and Hogeweg 1994; reviewed by Dunbar 2002), but they have yet to gain widespread acceptance (Bryson, Ando, and Lehmann 2007). We stress that these are null models, which need to be used as tools to begin to understand the behavior of real animals, rather than as an actual descriptions of an animal’s behavior (Peck 2004).

The central elements of a spatially-explicit agent-based simulation model include a habitat in which various kinds of resources (e.g., depleting food patches, sleeping sites) and agents (e.g., individual model animals, predators) occupy particular locations (Figure 2). Such a model would begin by explicitly designating the distribution of food resources, as food necessarily drives travel patterns - i.e., even if every other factor was missing from the environment, animals would still need to move to feed. Based on the spatially-explicit descriptions of environmental variation, a set of model parameters or dimensions that capture this variation is developed. Multiple agents within the simulation are then given simple foraging rules (e.g., "move to the next available patch" or "move to the largest patch within a certain distance”), and the emergent spatial structuring of those individuals (association patterns) are assessed after the model is run for a number of steps. The model is then iterated many times, and the resulting grouping patterns of the agents is recorded for different values of the environmental parameters, generating a surface that reflects what association patterns are predicted to be associated with what combinations of environmental variables. Observed field data can then be examined for concordance with this model surface. If we start with realistic values for meaningful 
environmental parameters, and notice that the particular grouping patterns arise only in certain environments and not in others, we obtain testable predictions about the effect of the environment on grouping, and this provides a means of understanding deviations from a purely ecological explanation. For examples of the use of spatially-explicit agent-based simulations see electronic supplement E.

- Figure 2 about here -

As with traditional socioecological models, accurate empirical measures of environmental variables of interest (e.g., food distribution) are desirable to lend "realism" to a simulation, but one important advantage of a simulation-based approach is that it is possible to explore the effect of a range of values for any model parameter, even ones very difficult to measure because of observational limitations or the time frame needed for accurate measures (e.g., predation pressure or infanticide risk). Moreover, once a simple model has been constructed, additional environmental variables can be added or removed to explore their effect on the emergent spatial structuring. In sophisticated models, the various resources and agents in the simulation might themselves show temporal variation in their size (e.g., growing and shrinking in size to simulate food patches such as fruiting trees with ripening and then diminishing crops), location (e.g., moving in space according to behavioral rules to simulate mobile prey or predators), or state (e.g., cycling through varying stages of "receptivity" to simulate female reproductive state).

The power of the agent-based modeling approach lies in its versatility and potential for expansion in ways that are informed by observational studies. For example, although initial null models might not explicitly incorporate social factors (e.g., dominance or kinship relationships among different agents) or demographic factors (e.g., group size, population sex ratio, number of simultaneously receptive females), which are known to influence the degree of spatial cohesion among animals (Lehmann and Boesch 2004), the modeling approach is sufficiently versatile to accommodate the inclusion of these 
kinds of variables. This could be done, for example, by altering the behavioral rules that agents follow when they encounter one another (e.g., "retreat” when the other agent is a more dominant agent, "follow" when the other agent is in estrus) or by making the behavior of agents contingent on accumulated information about the set of agents previously encountered (e.g., “do X” when the sex ratio of encountered agents is male-biased and “do Y” when it is female biased). Indeed, research on other mammalian taxa suggests that broadening our perspective from purely resource-based models to more socio-reproductively based ones may have considerable application to understanding primate spatial and social associations. For example, it is clear that a variety of social factors are key determinants of grouping patterns in other social mammals - e.g., the risk of infanticide influences grouping in lions (Grinnell and McComb 1996; Packer and Pusey 1983), allomaternal care shapes the social associations of elephants (Lee 1987), and the importance of reproductive competition over females shapes male alliances among dolphins (Connor et al. 2000). The potential influence of these kinds of variables on primate associations and sociality could be explored by integrating them into agent-based simulations, which in turn could be used to develop new predictions that could then be tested with field data. We believe that this integration of theory, modeling, and observation will be a profitable way to understand the causes and consequences of higher FF dynamics in primate and nonprimate taxa as well as to help identify the range of socioecological conditions under which lower FF groups are likely to arise.

\section{Implications of Fission-Fusion Dynamics for Communication}

${ }^{4}$ Karen B. Strier, Colleen M. Schaffner, Joseph H. Manson and Simon K. Bearder

${ }^{4}$ These authors have principal responsibility for this section 
Within primate groups, social signals may perform functions as simple as coordinating group movement (Boinski 1996), or as intricate as conveying and negotiating relationship qualities and processes such as formal submission and dominance (Flack and de Waal 2004, 2007), reconciliation (Aureli and de Waal 2000), and trust in the context of coalition formation (Smuts and Watanabe 1990). The kinds of signals and the size of signal repertoires selected to fulfill these functions are expected to be affected by fission-fusion dynamics (Kummer 2002; Milton 2000). Members of lower FF groups must resolve the conflicts generated by relatively high levels of association and avoid social disintegration. Members of higher FF groups must have ways to re-establish relationships and resolve uncertainties (e.g., concerning alliances, dominance status and each individual's relationship to third parties) depending on the frequency and patterns of spatial and temporal separations (Barrett, Henzi and Dunbar 2003). The interacting effects of the spatiotemporal variation in grouping patterns (see “Redefining Fission-Fusion” above) and social dynamics determine whether communication functions primarily to solve the problems posed by relatively high levels of association or those posed by extended or frequent spatial separations, independent of the sensory anatomy and physiology that different modes of communication (e.g., visual, olfactory, auditory) require.

Differences in communication patterns may reflect distinct evolutionary routes that could have led to both higher and lower FF groups, and we begin our consideration of communication with a description of two possible routes. We then present a framework for the evolution of social signaling that illustrates the relative importance of signals for maintaining spatial cohesion, separating from other group members, resolving uncertainty due to low spatial cohesion when individuals reunite, and negotiating social interactions depending on the degree of fission-fusion dynamics and the evolutionary route. We finish by showing the utility of the framework for generating testable hypotheses about how communicatory signals might vary depending on the social complexity and level of cohesion underlying fission-fusion dynamics. Our framework for the evolution of social signaling is heuristic. It 
is meant to stimulate research that investigates the extent to which variation in fission-fusion dynamics influences communication as little such data exist at present.

- Figure 3 about here -

\section{THE PHYLOGENY OF FISSION-FUSION DYNAMICS}

Figure 3 depicts two hypothetical evolutionary routes by which higher FF groups can arise and yield a variety of social systems (see electronic supplement B). On Route A, social relationships became increasingly valuable as the fitness gained through long-term associations - beginning between mates and/or between mothers and their adult offspring - increased. Along Route A, then, the driving force behind increasing gregariousness was the increasing value of social relationships for mutual advantages, which over time and with expanding scope could have resulted in lower FF groups, such as those of macaques and capuchin monkeys. The high value of social relationships (Cords 1997; Kummer 1978) selected for increasingly sophisticated relationship negotiation skills, thus permitting group members to remain together unless ecological pressures impose high levels of resource competition that necessitate fissioning. On Route A we can envision two main ways in which higher FF groups could have evolved: 1) by large groups fissioning into temporary smaller parties such as those observed in the communities of chimpanzees and spider monkeys (Nishida and Hiraiwa-Hasegawa 1987; Symington 1990); or 2) by small groups temporarily fusing into larger bands/clans and then aggregating with other bands/clans to exploit limited safe sleeping locations as is the case with the multilevel societies of hamadryas baboons (Stammbach 1987), with the basic subunits having a relatively fixed composition. On Route A, then, higher FF groups would have emerged among taxa in which sophisticated relationship negotiation skills already existed and formed the background from which the additional skills necessary for resolving relationship uncertainties in the face of periodic separations must have evolved. This scenario also conforms to the perception of higher FF taxa 
evolving along Route A having greater social complexity because their communication repertoire requires adeptness at negotiating social relationships while together, signaling departures, and reassessing their relationships following separation (Barrett et al. 2003; Dunbar 2003). The particular signals employed by higher FF groups need not be more cognitively demanding, but their repertoires may be more extensive than those of lower FF groups.

By contrast, species on Route B in our scenario would move to higher FF groups from a solitary rather than a group-living condition (Müller and Thalmann 2000; Sterline, Nguyen and Fashing 2000; Figure 3). Route B does not require that differentiated social relationships became increasingly valuable as gregariousness intensified, and associations may have been short-lived and random or opportunistic. In some galagos, for example, it may be thermally advantageous or safer with respect to predators to sleep with conspecifics than alone, but it is the number of conspecifics (as opposed to differences in their individual value as social partners) that leads them to fusion. However, it may also be easier or more efficient to associate with familiar conspecifics than with strangers, and the resulting higher FF groups may shift into facultatively lower FF groups if ecological conditions permit (Bearder 1999; Figure 3). Along Route B, the transition from solitary to higher FF groups could have required that minimal social skills (i.e., for distinguishing familiar individuals from strangers) and signals for resolving relationship uncertainties over distance and time became somewhat enhanced, though not to the level attained by lineages traversing Route A. Similarly, lower FF groups that emerge through Route B are expected to lack the range of relationship negotiation signals found in both lower FF and higher FF groups on Route A.

- Figure 4 about here -

MAPPING THE SOCIAL LANDSCAPE 
The different evolutionary trajectories by which higher FF groups could have arisen have implications for the evolution of social signaling. Figure 4 illustrates our framework by depicting a two-dimensional landscape for mapping social systems along two intersecting continua to consider the range and types of signaling mechanisms required to regulate spatial cohesion and social relationships. The X-axis in our landscape represents the degree of cohesiveness, operationalized here as the percentage of conspecifics within a given individual's social group that it can communicate with at any given time (which depends on the temporal variation in spatial cohesion, party size and composition; see electronic supplement C). We hypothesize that different kinds of signals are needed depending on whether they function primarily (a) to resolve relationship uncertainties that arise without constant contact and to convey information about fission events, or (b) to maintain coordination within relatively cohesive groups. The Y-axis of the landscape represents the complexity and differentiation of dyadic social relationships (sensu Hinde 1979), measurable by criteria such as high between-dyad variation and low within-dyad variation in rates and intensities of friendly and antagonistic behavior. At one extreme of our Y-axis dimension, adults would regard adult conspecifics of the same sex, reproductive condition and possibly degree of relatedness as interchangeable; at the other extreme, each dyadic relationship would be highly distinct along several dimensions (e.g., agonistic asymmetry, tolerance of close proximity, services provided). The X-axis demarcates a critical threshold for distinguishing between social environments that either do (upper) or do not (lower) require communication signals for the long-term maintenance of differentiated social relationships. Individuals of species that fall below this threshold may be able to recognize one another and may have signals for mediating their social interactions and coordinating their movements, but it is only among species that fall above this threshold that we expect to find signals that allow for the establishment and maintenance of long-term, differentiated social relationships.

The intersection of the two axes in Figure 4 divides the social landscape into four quadrants. Species in quadrant I are low in spatial cohesion and social complexity. Examples include solitary 
mammals in which adults associate only to mate and to contest access to resources (e.g., shrews: Cantoni 1993; giant mouse lemurs: Schülke and Ostner 2005). Courtship and assessment signals are used in these contexts, but they communicate only about the current interaction, not the dyadic relationship. Among species in quadrant II, signals serve to coordinate activities and maintain cohesive spatial associations, but social interactions are not based on differentiated social relationships. Examples include schooling fish that execute sophisticated anti-predator maneuvers (Pitcher and Wyche 1983).

In the upper half of Figure 4 we find animals that live in complex societies, which have been defined by de Waal and Tyack (2003a), as societies that are (a) individualized, (b) longitudinally stable, and probably (c) characterized by strong learning effects on social behavior and survival strategies. It is only in these societies that we expect to find signals by which individuals can convey the relationship qualities and processes described at the beginning of this section. We expect the evolution of communicative abilities to differ among taxa that fall in quadrants III and IV mainly because of differences in the degree to which individuals need to negotiate conflicts of interest immediately when extended separation is not a common option (quadrant III) or need to resolve uncertainties about relationships in the face of frequent separations (quadrant IV). We suggest that when fissioning is a viable ecological option, conflicts can be avoided instead of deliberately resolved. Prolonged, elaborate displays may be warranted during reunions when fissioning is rare (quadrant III), whereas simple greeting interactions of shorter duration may suffice when fission-fusion dynamics are common (quadrant IV) (Aureli and Schaffner 2007). Finally, different degrees of social complexity and sophistication in signaling should occur depending on whether species arrived in quadrant IV via Route A or Route B. Individuals from Route B (e.g., lesser galagos) would have a more limited range of signals than individuals from Route A (e.g., chimpanzees).

\section{ADDITIONAL FACTORS}


Local ecological conditions can result in interspecific variation that affects the relative placement of taxa within quadrants III or IV. We identify two parallel gradients in our qualitative assessment of primates (dotted lines in Figure 4), each of which reflects the range spanned by taxa with evolutionarily distinct life histories. The upper gradient represents taxa with comparatively slow life histories, such as the atelins, apes, capuchin monkeys and several Old World monkeys, while the lower gradient represents taxa with comparatively fast life histories, such as galagos and callitrichids. Taxa with slower life histories tend to have more complex and differentiated social relationships, based on their slower rates of development and longer life spans (Charnov 1991), than those with faster life histories when their degree of cohesiveness is similar.

Our landscape accounts for the differences in how signals are employed by populations of the same or closely-related species whose cohesion and quality of social relationships affect their respective positions in any of the quadrants. For example, the specialized reconciliation gestures observed in some captive chimpanzees (de Waal and Roosmalen 1979) are apparently absent in wild populations (Arnold and Whiten 2001), possibly because the increased spatial cohesion imposed by captivity necessitates more explicit, unambiguous reconciliation signals than is required when spatial separation through fission is an option. The gradients, which express the interacting effects of ecology and phylogeny, provide an additional basis for predictions about how intraspecific variation in signals might vary with a taxon's life history pattern. An additional factor affecting signaling is an individual's range of social opportunities (see electronic supplement F).

This framework for the evolution of social signaling generates a suite of testable hypotheses about the minimum requirements that different kinds of social patterns impose on communication. We have focused on the functions rather than the forms of social signals. However, consideration of the ways that different modes of communication permit individuals to keep track of one another without maintaining spatial cohesion is a critical next step (Kummer 2002; see electronic supplement G for examples). The parallel gradients that distinguish species according to their life history patterns, the 
impact of variation in social opportunities (see electronic supplement F), the evolutionary route followed to reach their position on the multi-dimensional fission-fusion space, and the different abilities to coordinate their activities over space and time provide additional variables around which future comparative studies on the role of communication in regulating social relationships can be designed.

\section{Cognitive Demands of Fission-Fusion Dynamics}

${ }^{5}$ Filippo Aureli, Josep Call, Richard Connor and Kay Holekamp

${ }^{5}$ These authors (in alphabetic order) have principal responsibility for this section

Chimpanzees, elephants, and dolphins - all taxa characterized by a high degree of fission-fusion dynamics - are also all species renowned for having relatively large brains and advanced cognitive abilities. One then might argue that possessing these characteristics and living in higher FF groups fit together in a causal manner. However, this is not necessarily true. Here, we explore this issue and propose a framework to identify some of the cognitive abilities we expect to find enhanced among animals living in higher FF groups.

\section{EVOLUTIONARY HISTORY MATTERS}

Chimpanzees, elephants, and dolphins are certainly special in that they display an array of cognitive abilities that are not found in many other animals, one of which is the apparently sophisticated manner in which they manage their social relationships over both space and time (de Waal and Tyack 2003b). Chimpanzees, elephants, and dolphins each have a particular evolutionary history and share a number of characteristics (e.g., slow life histories and long developmental periods), any and all of which may 
have influenced their cognitive abilities. Living in higher FF groups, therefore, may not be a causal factor, but simply a correlate of enhanced brain power.

Even if living in higher FF groups has evolutionarily consequences, its effects may well be contingent on what evolution was given to work with in the first place. When considering the whole array of species living in higher FF groups, as in the case of communication discussed above, we need to recognize the variation in cognitive abilities among these species, which is strongly dependent on the phylogenetic route by which a social organization with a higher degree of fission-fusion dynamics was achieved (see “The Phylogeny of Fission-Fusion Dynamics” above). For example, the ancestors of modern chimpanzees were likely group-living primates with the cognitive abilities to weigh the costs and benefits of interacting with multiple group members in ways dependent on current circumstances (Route A in Figure 3). This capacity could then be used in the fission-fusion context characterized by broader temporal and spatial scales. In this respect, chimpanzees stand in contrast to some other primates, like galagos, which have moved during their evolutionary histories from a solitary state towards a more social state in higher FF groups (Route B in Figure 3; Bearder 1999). In these cases, it is not a matter of employing an already existing set of social skills in a new context, but of developing them de novo. We should not assume, therefore, that a higher degree of fission-fusion dynamics automatically endows each species with the same cognitive abilities. Rather, given that a high degree of fission-fusion dynamics evolved multiple times in a wide range of taxa (from fish to birds to mammals), we should expect that cognitive adaptations to such dynamics are both variable and strongly influenced by the species’ evolutionary history, in addition to the nature of fission-fusion dynamics (see electronic supplements B) and the degree of differentiation of social relationships (from simple aggregations to complex societies: Figure 4). Comparative studies of cognitive adaptations to different degrees of fission-fusion dynamics should therefore use appropriate phylogenetic methods (e.g., Pagel and Harvey 1991; Nunn and Barton 2001). 


\section{THE DEMANDS OF SOCIO-SPATIAL FLEXIBILITY}

Another important issue is whether species living in higher FF groups have greater cognitive abilities than species that share a common evolutionary history but live in lower FF groups. We focus here on taxa that derive from group-living ancestors (Route A in Figure 3). When comparing species living in higher and lower FF groups, we must recognize that each kind of group offers opportunities for new skills to develop but also for certain selection pressures to be relaxed. We should not assume therefore that one kind of group necessarily produces greater cognitive abilities or "social intelligence" than another. With this in mind, we can formulate a framework regarding the cognitive demands that the socio-spatial flexibility of different fission-fusion dynamics may impose, and use this to develop testable hypotheses.

The degree of fission-fusion dynamics experienced may make different demands on the various cognitive processes involved in the acquisition, storage, processing, and use of social information. At present, it is not clear whether any cognitive abilities are unique to higher FF taxa, although various suggestions have been made (Barrett et al. 2003; Boesch and Boesch-Achermann 2000; Dunbar 2003; Milton 2000; Skoyles and Sagan 2002), and it seems most parsimonious to assume that we are dealing with relative enhancement of certain cognitive abilities, rather than a suite of new skills.

Living in lower FF groups may impose higher demands on information acquisition than living in higher FF groups because normally there are more group members present (and for longer periods) in the former than in the latter. Thus, members of lower FF groups are constantly bombarded with social information that often involves multiple partners engaged in fast-paced interactions. It is even possible that members of lower FF groups cope with such massive amounts of social information by paying selective attention to certain stimuli and filtering out redundant or unnecessary information. By contrast, individuals living in higher FF groups may display an enhanced ability to pick up subtle social cues and to use behaviors designed to probe others and extract relevant information from them (e.g., behaviors designed to test bonds: Zahavi 1977). These enhanced information acquisition skills may 
allow individuals to detect altered relationships among individuals who were temporarily in a different party (Connor 2007). Within higher FF taxa, these abilities are likely more important where party composition is highly variable (e.g., chimpanzees) and less relevant where the majority of social interactions are exchanged within parties with mostly fixed composition, although such parties fission and fuse with others to form multilevel societies (e.g., hamadryas baboons).

The storage and processing of information appear more demanding in higher FF than lower FF groups. This is a direct consequence of the patchier information gathered by individuals in higher FF groups. At the most basic level, these individuals must remember for longer periods the members of their own group because they are not exposed to them continuously. Moreover, due to changing party composition, these individuals not only have to keep track of who was involved in a particular interaction, but also the identity of others that were present (and absent) at the event. There is some evidence suggesting that chimpanzees can encode who witnessed an event such as a particular foodbaiting episode (Hare, Call and Tomasello 2001), but comparative systematic data are needed for appropriate testing of this hypothesis (and the other hypotheses below).

The dispersed nature of higher FF groups in space and time may have favored an ability to encode information concerning who was involved in what interaction and to remember such knowledge over longer time-frames than would be needed in lower FF groups. Renegotiating important long-standing relationships (sensu Kummer 1978), because individuals have forgotten where they stand with respect to each other, is wasteful of both time and energy. Selection should therefore favor individuals living in higher FF groups who are able to retain knowledge about partners (and their relationships with others, i.e., third party relationships) over a long time-scale, even in the absence of those partners, so they can “pick up where they left off” when they meet again.

The patchy nature of information gathered by members of higher FF groups may also promote enhancement of various information-processing skills. Inferential skills may enable animals to extract information about relationships among others from cues based on limited dyadic and triadic 
observations, which may be particularly important when all interactions cannot be witnessed. For example, spotted hyenas use transitive inference very effectively in social situations (Engh, Siebert, Greenberg and Holekamp 2005). Thus, although transitive inference is not exclusive to higher FF taxa (e.g., pinyon jays: Paz-y-Mino, Bond, Kamil and Balda 2004), we predict its enhancement in higher FF taxa.

It is also possible that, for some higher FF taxa, the ability to understand relations between relations (analogical reasoning) will be enhanced. This can act as a way of reducing cognitive demands while enhancing cognitive capacity (for example, by storing information on particular kinds of relationships, rather than retaining knowledge of many specific individual interactions: Call 2001). Within lower FF taxa, the need to store such information “off-line” may be reduced by the increased likelihood of witnessing interactions between dyads. Furthermore, in taxa where fission-fusion dynamics involve parties of mostly fixed composition (e.g., the joining and splitting of stable one-male units of hamadryas baboons), cognitive demands can be reduced by forming equivalence classes (cf. Schusterman, Reichmuth Kastak and Kastak 2003). For example, it would be easier to remember that “party X” was present during an interaction instead of individuals A, B, C, D and E.

Cognitive demands can also be reduced if assessment and updating of social relationships with various partners is achieved via emotional mediation, which is based on the emotion experienced when partners interact and the role of partner-dependent emotional experience in guiding future interactions (Aureli and Schaffner 2002). Under this scenario, individuals’ behavior toward other animals may reflect an emotional response akin to Damasio’s (1994) “somatic markers”, which guide current action by producing a positive or negative "gut feeling” about another individual, based on both experienced and observed interactions involving the other individual. This emotional response reflects the nature of past interactions but does not require any active recollection of them, or indeed any conscious cognitive response at all, merely a linking of a particular individual and situation with a particular emotional experience. Compared to lower FF taxa, emotional mediation in higher FF taxa should be based on a 
lower sample size of direct observations (because they do not witness all interactions of others) and on more indirect evidence (e.g., detectable changes in the behavior of others toward a particular individual after fusion).

Finally, information should be put to good use, and we can also hypothesize possible differences in this regard between higher FF and lower FF taxa. Owing to the changing opportunities of social partners in higher FF taxa, individuals may possess an enhanced capacity to inhibit and control their responses. For example, given that the appropriate response to a situation may vary depending on party composition, the capacity to inhibit responses under less favorable conditions should prove selectively advantageous. Thus, inhibiting prepotent responses, assessing a situation before acting, and possibly concealing changed relationships at reunions would all be expected to be enhanced in taxa experiencing a higher degree of fission-fusion dynamics. Japanese macaques provide an illustrative example of how members of lower FF groups may react to sudden changes in group composition without showing appropriate behavioral inhibition. High-ranking juvenile macaques whose relatives have been removed from the group invariably continued to challenge subordinate animals with intact matrilines (Chapais 1992). This led to the high-ranking juveniles losing their dominance rank due to the lack of support from their absent relatives. Chimpanzees, in contrast, seem to be able to inhibit aggressive behavior under challenging conditions (Aureli and de Waal 1997). As mentioned above, comparative systematic data are needed for appropriate testing of this and other hypotheses.

The particularly fluid nature of higher FF groups also may allow individuals to model the future and optimize social outcomes in many situations. For these individuals enhanced planning skills could be especially beneficial by enabling them to “engineer” party composition and associate with the most profitable partners (see below). Chimpanzees seem to display such planning skills during boundary patrols, territorial incursions and cooperative hunting (Boesch and Boesch-Achermann 2000; Wrangham 1999). There is also some indication of planning for future needs from tool use experiments in bonobos and orangutans (Mulcahy and Call 2006) and from the selection of the most helpful partners 
in a cooperative task in chimpanzees (Melis, Hare and Tomasello 2006). In addition, further cognitive demands can be encountered when individuals are in parties of varying size and composition due to a biological market of exchanges (Barrett et al. 2003; see electronic supplement H).

In conclusion, if different degrees of fission-fusion dynamics make characteristic cognitive demands on animals, then we should expect to see enhanced abilities of the kind described above in higher FF taxa compared to closely-related taxa living in lower FF groups. Empirical evidence for such a difference in demands and enhanced abilities is clearly needed. Our framework provides guidance on how to gather such evidence and how to carry out "fair" tests to probe these abilities. That is, speciesappropriate tests should be implemented to reflect the potentially different phylogenetic routes to fission-fusion dynamics and their different underlying brain substrates.

\title{
Implications of Fission-Fusion Dynamics for the Evolution of Complex Human Societies
}

\author{
${ }^{6}$ Amanda H. Korstjens, Robert Layton, Carel P. van Schaik, Christophe Boesch and Robin I. M. \\ Dunbar \\ ${ }^{6}$ These authors have principal responsibility for this section
}

Modern humans (and particularly modern foraging peoples: Marlowe 2005) offer what is in many ways an archetypal example of a higher FF taxon. We therefore build on issues raised in the preceding sections to investigate how human society - characterized by well-structured social relationships but with a low level of spatial cohesiveness during the day - may have evolved. In trying to understand the origins and evolution of human society, we can usefully ask four separate questions: (1) To what extent does human society differ from that seen in other anthropoid primates (in particular, in chimpanzees)? (2) By what evolutionary route did modern human social systems evolve? (3) What function(s) do 
higher FF groups have for hominids? And (4) what cognitive demands do higher FF groups impose on humans? We do not believe that it is possible to provide definitive answers to any of these questions at present; our intention, rather, is to develop a framework that identifies issues that need to be explored in order to provide those answers.

\section{HUMAN AND APE SOCIAL SYSTEMS COMPARED}

The social systems of great apes and humans share a low level of spatial cohesiveness but strongly developed social relationships (quadrant IV in Figure 4). Orangutans are clearly the least cohesive of the apes, but they are known to aggregate more when ecological conditions are favorable, and there appears to be some form of a community (Delgado and van Schaik 2000). Even the generally cohesive gorilla groups may be part of some form of multilevel society (Bradley, Doran-Sheehy, Lukas, Boesch and Vigilant 2004). Our closest relatives, chimpanzees and bonobos, whose physiology and food choice are more comparable to those of humans, have a social system with a high degree of fissionfusion dynamics but also a very strong level of cooperation and affiliation within communities. Bonobos differ from chimpanzees mainly in having slightly larger party sizes and a generally higher level of spatial cohesion. In addition, while male alliances are important in chimpanzees, female alliances and male-female alliances are more important in bonobos (Hohmann and Fruth 2002; Parish and de Waal 2000). For simplicity, we limit our framework mainly to a comparison between early humans and chimpanzees (including bonobos, unless stated otherwise).

Humans and chimpanzees share a number of features (see also Layton and Barton 2001; Moore 1996; Nishida and Hiraiwa-Hasegawa 1987): an omnivorous diet associated with cooperative hunting, tool use (though cooperative hunting and tool use may not apply to bonobos), large community size, and higher FF groups within the context of a multimale/multifemale social organization. There are also a number of shared features that are expressed differently in the two taxa: sexual division of labor (only in humans does this entail a form of mutual dependence), hunting (meat sharing occurs in both 
chimpanzees and humans, but only in humans do we see prey killed elsewhere and brought back to a camp for distribution), and flexible territoriality. To compare the differences between chimpanzees and modern humans regarding territorial tendencies, we first need to understand the phylogenetic connection between human and chimpanzee grouping patterns. If the human band (i.e., overnight camp) corresponds to the chimpanzee community, then territoriality is more relaxed in modern humans than in chimpanzees. In many hunter-gatherer societies, adult humans of both sexes can readily move (temporarily or permanently) between bands (e.g., Lee 1979: 42, Turnbull 1965: 96, Woodburn 1982: 435). However, if the regional hunter-gatherer community (commonly associated with a unique dialect) corresponds to chimpanzee community then territoriality of modern hunter-gatherers is more comparable to that of chimpanzees. Relations between regional communities are more constrained (e.g., Andrews 1996), although exchange across community boundaries does occasionally take place (e.g., McBryde 1978). When we assume that chimpanzee communities should be compared to regional communities (and the cognitive evidence suggests that this is the correct equivalence: Dunbar 1993), then human social evolution has moved toward longer-term association in sub-units (i.e., the time intervals between fusion of subunits has increased).

Finally, human societies differ from those of apes in a number of respects: the assembly of the whole band at an overnight base camp, the presence of social and economic pair bonds, significant alloparental inputs in child rearing, exchange of goods as tokens of social relations, and, perhaps rather obviously, the use of language in regulating relationships (see also Dunbar 1993; Marlowe 2005; Rodseth et al. 1991). These differences are numerically and qualitatively sufficiently large that they are unlikely to have arisen at the same time, which raises the second issue of our framework: how were these differences acquired in the hominid lineage?

PHYLOGENY AND FUNCTION FOR HUMAN HIGHER FF GROUPS 
At present, we can say little about the sequence of specific events that occurred in the hominid lineage, but it is likely that human higher FF groups derived from group-living species that depended on cooperation and had various social skills (e.g., conflict resolution: Aureli and de Waal 2000; alliance formation: Harcourt and de Waal 1992) useful for maintaining social relationships in spite of low spatial cohesion (Route A in Figure 3). The best we can do is to assume that whatever chimpanzee and bonobo societies have in common with those of modern humans resembles those of their common ancestor (Moore 1996) and then ask what changes had to be made, and in what sequence, in order for the differences to develop. Because these changes are inevitably linked to the function of fissioning and fusing, the third question of our framework is embedded in the discussion below.

Most of the differences we listed between chimpanzee and human society are unique features of humans and probably evolved fairly recently in human evolutionary history. The use of sites where the community aggregates at night (i.e., a possible precursor of home bases), however, is common in many primates (although not in chimpanzees or bonobos). The use of a limited number of sleeping sites is driven by predation pressure and availability of safe refuges that are large enough for the group (Anderson 2000). If the society has a relatively low level of spatial cohesiveness, a lack of safe sleeping sites or extreme predation risk may force the group members to come together at night. Such a situation is especially obvious in hamadryas baboons (Kummer 1995), though similar patterns may be seen in all Papio baboons. The fact that baboons live in as large a variety of habitats as our earliest ancestors did (from rainforests to open savannah areas), including more open and drier habitats than those where modern chimpanzees occur, seems relevant, given that Papio and our earliest ancestors first emerged at about the same time (Jolly 2001; Moore 1996). We envisage an evolutionary path in which a chimpanzee-like hominid ancestor, with a low degree of spatial cohesiveness but highly structured social relationships, started to exploit not just forests but also more open savannah/woodland environments. In such environments, daytime food searching demands required individuals to forage in small parties, just like contemporary chimpanzees in forested habitats and baboons in dry savanna 
habitats do (Nishida and Hiraiwa-Hasegawa 1987; Stammbach 1987). However, some variable - most likely the rarity of essential resources such as safe refuges at night (with an increased risk of predation in the more open habitat) or waterholes (scarce in savannah habitats but less so in forests) - forced individuals to aggregate once a day in large groups that contained the entire band or community, as happens in hamadryas baboons (Stammbach 1987), but unlike contemporary woodland and forest chimpanzees (Nishida and Hiraiwa-Hasegawa 1987).

Coming together at night and at waterholes is not the only difference between the fission-fusion dynamics of chimpanzees and baboons: they also differ in the type of parties that are commonly formed. As discussed earlier (see “Fission-Fusion Societies?” above), in hamadryas baboons large night aggregations split into smaller foraging parties based on one-male units during the course of the day, whereas in chimpanzees community members are very rarely all together and form parties rather independently. This difference can have a major impact on the level of cooperation that occurs among community members. A chimpanzee-like system may be more conducive to cooperation (as seen among chimpanzee males during hunting of difficult prey and border patrols) than a hamadryas baboon-like system because all members of a chimpanzee community may regularly interact affiliatively when they meet in different parties. In a baboon-like system, on the other hand, individuals always remain in the same party (at the smallest unit level) and there is little affiliative interaction between members of different parties.

We do not know what kind of aggregation or dispersal behavior may have occurred among australopithecines, but most paleoanthropologists agree that australopithecines were highly sexually dimorphic (similar to baboons) and that therefore neither a chimpanzee-like social system nor that approximating modern hunter-gatherers was found among them. Rather, inter-male competition may have been similarly strong as it is in baboons (Foley and Lee 1989; Plavcan and van Schaik 1997), which tends to reduce levels of cooperation among males. Indeed, what was once thought to be evidence for "home bases" among early Palaeolithic hominids is now discounted, as the accumulations 
of tools and bones are considered to have been caused by transport in streams or flash floods (Binford 1987; Stern 1993). All we can say at this stage is that the typical chimpanzee strategy of building nests wherever individuals happen to find themselves at nightfall would have been constrained by the occurrence of fewer suitable sites in more open savannah/woodland environments. We stress nonetheless that this hypothetical formation of large groups at least once a day at a safe location would, in combination with a society in which cooperation is common, offer a whole new set of opportunities, especially for a large-brained primate like our ancestors. Note that some of the benefits of aggregating once a day do not require anything as advanced as a home base, but only require that individuals spend a relatively long period of social time in each other's presence while not engaged in activities such as foraging, hunting, or patrolling their boundaries.

As the daily aggregation at a safe limiting resource evolved into a home base in later Homo species, fusing at nighttime may have offered the opportunity for sexual division of labor whereby gatherers and hunters shared their spoils at the end of the day (Key and Aiello 2000). Some degree of resource redistribution would be allowed by alloparenting (Kennedy 2003), costly signaling by males, and perhaps pair bonding; the latter could allow pooling of resources within a domestic unit in a context where confidence in reciprocity of food transfers was maximized (a feature that may have been reinforced or actively selected for by the parental investment demands of increasing brain size: Key and Aiello 2000). Because they no longer had to leave the safety of the home base to forage, the home base would presumably have increased the survival chances of the weakest individuals (e.g., young children, older people, women with newborns, and heavily pregnant women) who could nonetheless perform essential tasks at the home base such as childcare. At this stage, the sharing of spoils means that individuals in the community could distribute themselves optimally over the area to increase efficient use of resources. Eventually, this could have given rise to the multi-layered society of modern hunter-gatherers in which survival risks were further reduced through cooperation between different bands, in the form of trading and allowing some trespassing onto each other's territories (Klein 1999; 
Layton and Barton 2001). Many of these latter changes imply significant demands in terms of social skills and may have selected for the evolution of larger brains (see electronic supplement I for archeological evidence).

\section{COGNITIVE DEMANDS OF HUMAN HIGHER FF GROUPS}

This leads us into the last of the four questions of our framework, because the scale of human social groups (and, in particular, the size and dispersion of forager communities, in combination with a gathering at home bases) must inevitably impose considerable demands on human cognition (Aiello and Dunbar 1993). We would simply like to stress that a high degree of fission-fusion dynamics may be cognitively taxing (see “Cognitive Demands of Fission-Fusion Dynamics” above) and, without the gathering of individuals at night, may undermine high degrees of cooperation for two reasons. First, fission-fusion dynamics do not allow for regular updating of information about social relationships in the community and this makes it more difficult for individuals to depend too strongly on a relationship when individuals are spatially dispersed. Second, regular fissioning and fusing creates opportunities for free riders (Enquist and Leimar 1993) because each individual's behavior cannot be monitored while the group is dispersed. Coming together daily not only allows individuals to reinforce bonds, it provides opportunities to observe the social interactions taking place among other individuals (and thus update reputations) and additionally forces individuals to resolve conflicts in order to reduce tensions in the group instead of simply avoiding one another. In combination with the cognitive abilities of great apes and their tendency towards complex social relationships, this could have been the point of departure for the high level of cooperation and the evolution of complex symbols to indicate intentions that we observe in hominids. Social learning and information exchange at these daily gatherings is likely to further increase the development of a society with highly structured social relationships and elaborate signals. Even though the original need for fusing on a daily basis may have disappeared as hominids came to occupy various environments, the habit of doing so may just have been the 
evolutionary adaptation that allowed our ancestors to become a highly adaptable species, capable of spreading across the world.

\section{Conclusions}

The five interlinked sections of this article propose several new frameworks within which to pursue research on a range of topics that we believe are particularly relevant to fission-fusion dynamics. In the 30 years since the term "fission-fusion" was first introduced (Kummer 1971), research in this area has been rather patchy and has focused only on a limited number of topics. For example, significant methodological improvement for the recognition of non-random association patterns has been made (see electronic supplement A), and several socioecological factors influencing party size have been identified (e.g., Chapman et al. 1995; Lehmann and Boesch 2004; Mitani, Watts and Lwanga 2002). Less attention has been paid to other aspects of fission-fusion dynamics, such as the rate of change in party size or the extent to which parties, when they do form, are comprised of the same set of individuals.

A possible reason for the lack of more systematic investigation of fission-fusion dynamics is the tendency for researchers to continue to adhere to a dichotomous perspective on flexible versus cohesive organizations and to have mainly focused on the so-called "fission-fusion societies" of chimpanzees and spider monkeys. We believe that much progress can be achieved by acknowledging that this is a false dichotomy and by recognizing that fission-fusion dynamics occur to some extent in most social systems (cf. Kinzey and Cunningham 1994; Strier 1989; Struhsaker and Leland 1979; Sussman and Garber 2007). We reiterate, therefore, that a starting point for a renewed research input is the adoption of a relativistic approach that places species - and even populations of the same species - within a complex, multidimensional fission-fusion space of the sort illustrated in Figure 1. Higher and lower FF groups refer to relative positions within such a space, and comparisons between taxa and populations 
thus need to be interpreted within such a relativistic framework of higher or lower degrees of fissionfusion dynamics.

A second critical point we have stressed is the pressing need for primatologists - and other researchers interested in the evolution of flexible patterns of social organization - to develop new null models against which the associations of individuals can be compared. The generalized modeling framework of spatially-explicit agent-based simulation is a powerful tool that can be applied to many different taxa. Another critical point emphasized in our frameworks is that the evolutionary route followed to reach a given degree of fission-fusion dynamics has fundamental implications for the types of social and cognitive skills the organism may posses. This means that two species that lie relatively close to one another in the multidimensional fission-fusion space may nonetheless differ in important respects depending on their phylogenetic background. Thus, interspecific comparisons need to take evolutionary history into account (Pagel and Harvey 1991; Nunn and Barton 2001).

The relativistic approach based on the degree of fission-fusion dynamics within such an evolutionary framework provides an effective way to evaluate patterns of communication across taxa. Given similar differentiation of social relationships, we expect that communication in lower FF groups would be focused around coordinating movement, resolving conflicts and promoting cooperation, whereas the communication systems of higher FF groups must accommodate the additional requirements of routinely negotiating separations, re-establishing relationships, and reducing tension at reunions. In addition, while carrying out comparative analyses we need to be aware that higher FF groups may have evolved either from rather solitary species with limited social skills or from relatively cohesive groups that already possessed effective means for maintaining differentiated relationships. Furthermore, despite the fact that many animals living in higher FF groups have large brains and sophisticated cognitive abilities, we suggest that a higher degree of fission-fusion dynamics does not necessarily impose selection pressures that promote the evolution of novel forms of "social intelligence”. However, evolutionary enhancement in existing cognitive skills may increase fitness in 
higher FF taxa, and thus promote cognitive attributes such as long-term memory about who did what to whom and the inferential abilities necessary to put together patchy social information. Finally, the nature of fission-fusion dynamics of early hominids, in particular the suggested combination of both a high degree of interaction among community members due to frequent changes in party composition and a tendency to reunite on a daily basis, may have strongly influenced the evolution of the highly adaptable and cognitively sophisticated species that we are today.

Given these premises, the proposed frameworks we advocate here are expected to stimulate new momentum in the investigation of whether qualitative or quantitative differences in fact exist between species or populations in the underlying socioecological conditions, communication skills, or required cognitive abilities depending on their degree of fission-fusion dynamics. As higher FF groups occur in a wide variety of taxa, a broad comparative perspective, not limited to primates, should help elucidate underlying principles, and also highlight fundamental differences due to evolutionary history. This perspective could also be highly beneficial in increasing our understanding of the causes of the variability in human fission-fusion dynamics and the evolution of our complex societies. 


\section{References Cited}

Aiello, L. C., and R. I. M. Dunbar. 1993. Neocortex size, group size, and the evolution of language. Current Anthropology 34:184-193.

Anderson, J. R. 2000. Sleep-related behavioural adaptations in free-ranging anthropoid primates. Sleep Medicine Reviews 4:355-373.

Andrews, E. 1996. Territory and land use among the Akulmiut of Western Alaska. In Key issues in hunter-gatherer research, ed. E. Burch and L. Ellanna, 65-93. Oxford: Berg.

Arnold, K., and A. Whiten. 2001. Post-conflict behavior of wild chimpanzees (Pan troglodytes schweinfurthii) in the Budongo Forest, Uganda. Behaviour 138:649-690.

Aureli, F., and F. B. M. de Waal. 1997. Inhibition of social behavior in chimpanzees under high-density conditions. American Journal of Primatology 41:213-228.

Aureli, F., and F. B. M. de Waal. 2000. Natural conflict resolution. Berkeley: University of California Press.

Aureli, F., and C. M. Schaffner. 2002. Relationship assessment through emotional mediation. Behaviour 139:393-420.

Aureli, F., and C. M. Schaffner. 2007. Aggression and conflict management at fusion in spider monkeys. Biology Letters 3:147-149.

Barrett, L., P. Henzi, and R. Dunbar. 2003. Primate cognition: From 'what now?’ to ‘what if?’. Trends in Cognitive Science 7:494-497.

Bearder, S. K. 1999. Physical and social diversity among nocturnal primates: a new view based on long term research. Primates 40:67-282.

Binford, L. R. 1987. Searching for camps and missing the evidence? Another look at the Lower Palaeolithic. In The pleistocene old world, ed. O. Soffer, 17-32. New York: Academic Press. 
Boesch, C., and H. Boesch-Achermann. 2000. The chimpanzees of the Taï forest. Oxford: Oxford University Press.

Boinski, S. 1996. Vocal coordination of troop movement in squirrel monkeys (Saimiri oerstedi and S. sciureus) and white-faced capuchins (Cebus capucinus). In Adaptive radiations of Neotropical primates, ed. M.A. Norconk, A.L. Rosenberger, and P.A. Garber, 251-69. New York: Plenum Press.

Boyer, D., O. Miramontes, G. Ramos-Fernández, J. L. Mateos, G. Cocho, and H. Larralde. 2004. Modeling the searching behavior of social monkeys. Physica A: Statistical Mechanics and its Applications 342:329-335.

Boyer, D., G. Ramos-Fernández, O. Miramontes, J. L. Mateos, G. Cocho, H. Larralde, H. Ramos, and F. Rojas. 2006. Scale-free foraging by primates emerges from their interaction with a complex environment. Proceedings of the Royal Society of London Series B-Biological Sciences doi: 10.1098/rspb.2005.3462

Bradley, B. J., D. M. Doran-Sheehy, D. Lukas, C. Boesch, and L. Vigilant. 2004. Dispersed male networks in western gorillas. Current Biology 14:510-513.

Bryson, J. J., Y. Ando, and H. Lehmann. 2007. Agent-based modelling as scientific method: A case study analysing primate social behaviour. Philosophical Transactions of the Royal Society of London, B 362:1685-1698.

Call, J. 2001. Chimpanzee social cognition. Trends in Cognitive Sciences 5:388-393

Cantoni, D. 1993. Social and spatial organization of free-ranging shrews, Sorex coronaturs and Neomys fodiens, (Insectivora, Mammalia). Animal Behaviour 45:975-995.

Chapais, B. 1992. The role of alliances in social inheritance of rank among female primates. In Coalitions and alliances in human and nonhuman animals, ed. A. H. Harcourt and F. B. M. de Waal, 29-59. New York: Oxford University Press. 
Chapman, C. A., R. W. Wrangham, and L. J. Chapman. 1995. Ecological constraints on group size: an analysis of spider monkey and chimpanzee subgroups. Behavioral Ecology and Sociobiology 36:59-70.

Charnov, E. L. 1991. Evolution of life history variation among female mammals. Proceedings of the National Academy of Sciences USA 88:1134-1137.

Connor, R. C. 2007. Complex alliance relationships in bottlenose dolphins and a consideration of selective environments for extreme brain size evolution in mammals. Philosophical Transactions of the Royal Society B 362:587-602.

Connor, R. C., R. Wells, J. Mann, and A. Read. 2000. The bottlenose dolphin, Tursiops spp.: Social relationships in a fission-fusion society. In Cetacean societies: Field studies of whales and dolphins, ed. J. Mann, R. C. Connor, P. Tyack, and H. Whitehead, 91-126. Chicago: The University of Chicago Press.

Cords, M. 1997. Friendships, alliances, reciprocity and repair. In Machiavellian intelligence II: extensions and evaluations, ed. A. Whiten, and R. W. Byrne, 24-49. Cambridge, UK: Cambridge University Press

Dale, M. R. T., P. Dixon, M.-J. Fortin, P. Legendre, D. E. Myers, and M. S. Rosenberg. 2002. Conceptual and mathematical relationships among methods for spatial analysis. Ecography 25:558577.

Damasio, A.R. 2004. Descartes’ error: emotion, reason and the human brain. New York: Grosset/Putnam.

Delgado, R. A., Jr., and C. P. van Schaik. 2000. The behavioral ecology and conservation of the orangutan (Pongo pygmaeus): a tale of two islands. Evolutionary Anthropology 9:201-218. de Waal, F. B. M., and A. Roosmalen. 1979. Reconciliatiiton and consolation among chimpanzees. Behavioral Ecology and Sociobiology 5:55-66. 
de Waal, F. B. M., and P. L. Tyack. 2003a. Preface. In Animal social complexity - intelligence, culture, and individualized societies, ed. F. B. M. de Waal and P. L. Tyack, ix-xiv. Cambridge, MA: Harvard University Press.

de Waal, F. B. M., and P. L. Tyack. 2003b. Animal social complexity - intelligence, culture, and individualized societies. Cambridge, MA: Harvard University Press.

Dunbar, R. I. M. 1988. Primate social systems. Ithaca, NY: Cornell University Press.

—. 1993. Coevolution of neocortical size, group size, and language in humans. Behavior and Brain Sciences 16:681-735.

—. 2002. Modeling primate behavioral ecology. International Journal of Primatology 24:785-819.

—. 2003. Why are apes so smart? In Primate life histories and socioecology, ed. P. M. Kappeler and M. E. Pereira, 285-98. Chicago: University of Chicago Press.

Engh, A. L, E. R. Siebert, D. A. Greenberg, and K. E. Holekamp. 2005. Patterns of alliance formation and post-conflict aggression indicate spotted hyaenas recognize third party relationships. Animal Behaviour 69:209-217.

Enquist, M., and O. Leimar. 1993. The evolution of cooperation in mobile organisms. Animal Behaviour 45:747-757.

Flack, J. C., and F. B. M. de Waal. 2004. Dominance style, social power, and conflict management. In Macaque societies: A model for the study of social organization, ed. B. Thierry, M. Singh, and W. Kaumanns, 157-85. Cambridge: Cambridge University Press.

_ - 2007. Context modulates signal meaning in primate communication. Proceedings of the National Academy of Sciences 104:1581-1586.

Foley, R., and P. C. Lee. 1989. Finite social space, evolutionary pathways, and reconstructing hominid behaviour. Science 243:901-906. 
Fortin, M.-J., M. R. T. Dale, and J. van Hoef. 2002. Spatial analysis in ecology. In Encyclopedia of environmetrics, vol. 4, ed. A. H. El-Shaarawi and W. W. Piegorsch, 2051-2058. Chichester: John Wiley \& Sons, Ltd.

Ghiglieri, M. P. 1984. The chimpanzees of the Kibale forest: A field study of ecology and social structure. New York: Columbia University Press.

Grinnell, J., and K. McComb. 1996. Maternal grouping as a defense against infanticide by males: evidence from field playback experiments on African lions. Behavioral Ecology 7:55-59.

Harcourt, A. H., and F. B. M. de Waal. 1992. Coalition and alliances in humans and other animals. Oxford: Oxford University Press.

Hare, B., J. Call, and M. Tomasello. 2001. Do chimpanzees know what conspecifics know and do not know? Animal Behaviour 61:139-151.

Henzi, P., and L. Barrett. 2003. Evolutionary ecology, sexual conflict, and behavioral differentiation among baboon populations. Evolutionary Anthropology 12:217-230.

Hinde, R. A. 1979. Towards understanding relationships. New York: Academic Press.

Hohmann, G., and B. Fruth. 2002. Dynamics in social organization of bonobos (Pan paniscus). In Behavioural diversity in chimpanzees and bonobos, ed. C. Boesch, G. Hohmann, and L. F. Marchant, 138-150. Cambridge: Cambridge University Press.

Holekamp, K. E., S. M. Cooper, C. I. Katona, N. A. Berry, L. G. Frank, and L. Smale. 1997. Patterns of association among female spotted hyenas (Crocuta crocuta). Journal of Mammalogy 78:55-64.

Holekamp, K., E. E. Boydston, and L. Smale. 2000. Group travel in social carnivores. In On the Move, ed. S. Boinski, and P. A. Garber, 587-627. Chicago: The University of Chicago Press.

Janson, C. H. 2000. Primate socio-ecology: the end of a golden age. Evolutionary Anthropology 9:7386.

Jolly, C. J. 2001. A proper study for mankind: analogies from the papionin monkeys and their implications for human evolution. Yearbook of Physical Anthropology 44:177-204. 
Kappeler, P. M., and C. P. van Schaik 2002. Evolution of primate social systems. International Journal of Primatology 23:707-740.

Kennedy, G. E. 2003. Palaeolithic grandmothers? Life history theory and early Homo. Journal of the Royal Anthropological Institute 9:549-572.

Kerth, G., and B. König. 1999. Fission, fusion and nonrandom association in female Bechstein’s bats (Myotis bechsteinii). Behaviour 136:1187-1202.

Key, C., and L. C. Aiello. 2000. A prisoner's dilemma model of the evolution of paternal care. Folia Primatologica 71:77-92.

Kinzey, W.G., and E. P. Cunningham. 1994. Variability in platyrrhine social organization. American Journal of Primatology 34:185-198.

Klein, R. G. 1999. The human career. Chicago: The University of Chicago Press.

Klein, L. L., and D. J. Klein. 1977. Feeding behavior of the Colombian spider monkey, Ateles belzebuth. In Primate ecology: Studies of feeding and ranging behaviour in lemurs, monkeys, and apes, ed. T. H. Clutton-Brock, 153-181. London: Academic Press.

Kummer, H. 1968. Social organization of hamadryas baboons. Chicago: University of Chicago Press.

—. 1971. Primate societies - group techniques of ecological adaptation. Chicago: Aldine Publishing Company.

—. 1978. On the value of social relationships to nonhuman primates: A heuristic scheme. Social Science Information 17:687-705.

—. 1995. In quest of the sacred baboon. Princeton: Princeton University Press.

—. 2002. Topics gained and lost in primate social behavior. Evolutionary Anthropology 11(Suppl 1):73-74.

Layton, R., and R. A. Barton. 2001. Warfare and human social evolution. In Ethnoarcheology of hunter-gatherers: pictures at an exhibition, BAR International Series 955, ed. K. J. Fewster and M. Zvelebil, 13-24. Oxford: Archaeopress. 
Lee, P. C. 1987. Allomothering among African elephants. Animal Behaviour 35:278-291.

—. 1994. Social structure and evolution. In Behaviour and evolution, ed. P. Slater and T. Halliday, 266-303. Cambridge: Cambridge University Press.

Lee, R.B. 1979. The !Kung San: men, women and work in a foraging society. Cambridge: Cambridge University Press.

Lehmann, J., and C. Boesch. 2004. To fission or to fusion: effects of community size on wild chimpanzee (Pan troglodytes verus) social organisation. Behavioral Ecology and Sociobiology 56:207-216.

Marlowe, F. W. 2005. Hunter-gatherers and human evolution. Evolutionary Anthropology 14:54-67.

McBryde, I. 1978. Wil-im-ee Moor-ing: or, Where do Axes come from? Mankind 11:328-54.

Melis, A. P., B. Hare, and M. Tomasello. 2006. Chimpanzees recruit the best collaborators. Science 311:1297-1300.

Milton, K. 1984. Habitat, diet, and activity patterns of free-ranging woolly spider monkeys (Brachyteles arachnoides E Geoffroy 1806). International Journal of Primatology 5:491-514.

—. 2000. Quo vadis? Tatics of food search and group movement in primates and other animals. In On the move: How and why animals travel in groups, ed. S. Boinski and P. A. Garber, 375-417. Chicago: The University of Chicago Press.

Mitani, J. C., D. P. Watts, and M. N. Muller. 2002. Recent developments in the study of wild chimpanzee behaviour. Evolutionary Anthropology 11:9-25.

Mitani, J. C., D. P. Watts, and J. S. Lwanga. 2002. Ecological and social correlates of chimpanzee party size and composition. In Behavioural diversity in chimpanzees and bonobos, ed. C. Boesch, G. Hohmann, and L. F. Marchant, 102-111. Cambridge: Cambridge University Press.

Moore, J. 1996. Savanna chimpanzees, referential models and the last common ancestor. In Great ape societies, ed. W. C. McGrew, L. F. Marchant, and T. Nishida, 275-292. Cambridge: Cambridge University Press. 
Moss, C. J. , and P. C. Lee. in press. Female social dynamics: Fidelity and flexibility. In The Amboseli elephant: a long term perspective on a long lived species, ed. C. J. Moss and H. Croze. Chicago: The University of Chicago Press.

Mulcahy, N. J., and J. Call. 2006. Apes save tools for future use. Science 312:1038-1040.

Müller, A. E., and U. Thalmann. 2000. Origin and evolution of primate social organisation: a reconstruction. Biological Review of the Cambridge Philosophical Society 75:405-435.

Nishida, T., and M. Hiraiwa-Hasegawa. 1987. Chimpanzees and bonobos: cooperative relationships among males. In Primate societies, ed. B. B. Smuts, D. L. Cheney, R. M. Seyfarth, R. W. Wrangham and T. T. Struhsaker, 165-177. Chicago: The University of Chicago Press.

Nunn, C. L., and R. A. Barton. 2001 Comparative methods for studying primate adaptation and allometry. Evolutionary Anthropology 10:81-98.

Packer, C., and A. E. Pusey. 1983. Adaptations of female lions to infanticide by incoming males. The American Naturalist 121:716-728.

Pagel, P.H., and M. D. Harvey. 1991. The comparative method in evolutionary biology. Oxford: Oxford University Press

Parish, A. R., and F. B. M. de Waal. 2000. The other "closest living relative" - How bonobos (Pan paniscus) challenge traditional assumptions about females, dominance, intra- and intersexual interactions, and hominid evolution. Annals of the New York Academy of Sciences 907:97-113.

Plavcan, J. M., and C. P. van Schaik. 1997. Interpreting hominid behavior on the basis of sexual dimorphism. Journal of Human Evolution 32:345-374.

Paz-y-Mino, G., A. B. Bond, A. C. Kamil, and R. P. Balda. 2004. Pinyon jays use transitive inference to predict social dominance. Nature 430:778-781.

Peck, S. L. 2004. Simulation as experiment: a philosophical reassessment of biological modeling. Trends in Ecology and Evolution 19:530-534. 
Pitcher, T. J., and C. Wyche. 1983. Predator avoidance behaviour in sand-eel schools: why do schools seldom split? In Predators and prey in fishes, ed. D. Noakes, B. Lindquist, G. Helfman, and J. Ward, 193-204. The Hague: Junk.

Pusey, A. E. 1979. Intercommunity transfer of chimpanzees in Gombe National Park. In The great apes, ed. D. A. Hamburg and E. R. McCown, 465-480. Menlo Park, CA: Benjamin/Cummings. Pusey, A. E., and C. Packer. 1987. Dispersal and philopatry. In Primate societies, ed. B. B. Smuts, D. L. Cheney, R. M. Seyfarth, R. W. Wrangham, and T. T. Struhsaker, 250-266. Chicago: University of Chicago Press.

Ramos-Fernández, G., D. Boyer, and V. P. Gómez. 2006. A complex social structure with fissionfusion properties can emerge from a simple foraging model. Behavioral Ecology and Sociobiology 60:536-549.

Rodman, P. S., and A. Di Fiore. 1993. Effects of group size and resource dispersion on foraging efficiency of primates: A simulation model. American Journal of Physical Anthropology S16:166.

Rodseth, L., R. W. Wrangham, A. M. Harrigan, and B. B. Smuts. 1991. The human community as a primate society. Current Anthropology 32:221-254.

Schülke, O., and J. Ostner. 2005. Big times for dwarfs: social organization, sexual selection, and cooperation in the Cheirogaleidae. Evolutionary Anthropology 14:170-185.

Schusterman, R. J., C. Reichmuth Kastak, and D. Kastak. 2003. Equivalence classification as an approach to social knowledge: from sea lions to simians. In Animal social complexity - intelligence, culture, and individualized societies, ed. F. B. M de Waal and P. L. Tyack, 179-206. Cambridge, MA: Harvard University Press.

Skoyles, J.R., and Sagan D. 2002. Up from dragons: the evolution of human intelligence. New York, McGraw-Hill.

Smuts, B. B., and J. Watanabe. 1990. Social relationships and ritualized greetings in adult male baboons (Papio cynocephalus anibis). International Journal of Primatology 11:147-172. 
Smuts, B. B., D. L. Cheney, R.M. Seyfarth, R.W. Wrangham, and T.T. Struhsaker. 1987. Primate societies. Chicago: The University of Chicago Press.

Stammbach, E. 1987. Desert, forest, and montane baboons: Multilevel societies. In Primate societies, ed. B. B. Smuts, D. L. Cheney, R. M. Seyfarth, R. W. Wrangham, and T. T. Struhsaker, 112-120. Chicago: The University of Chicago Press.

Sterling, E., N. Nguyen, and P. Fashing. 2000. Spatial patterning in nocturnal prosimians: a review of methods and relevance to studies of sociality. American Journal of Primatology 51:3-19.

Stern, N. 1993. The structure of the lower pleistocene archaeological record: a case study from the Koobi Fora Formation. Current Anthropology 34:201-226.

Strier, K. B. 1989. Effects of patch size on feeding associations in muriquis (Brachyteles arachnoids). Folia Primatologica 52:70-77.

—. 1992. Atelinae adaptations: behavioral strategies and ecological constraints. American Journal of Physical Anthropology 88:515-524.

—. 1994. Brotherhoods among atelins: kinship, affiliation, and competition. Behaviour 130:151-167.

—. 2003. Primatology comes of age: 2002 AAPA luncheon address. Yearbook of Physical Anthropology 46:2-13.

Struhsaker, T. T., and L. Leland. 1979. Socioecology of five sympatric monkey species in the Kibale Forest, Uganda. Advances in the Study of Behavior 9:159-228.

Stumpf, R. 2007. Chimpanzees and bonobos: diversity within and between species. In Primates in perspective, ed. C. J. Campbell, A. Fuentes, K. C. MacKinnon, M. Panger, S. K. Bearder, 321-344. Oxford: Oxford University Press.

Sussman, R. W., and Garber, P. A. 2007. Cooperation and competition in primate social interactions. In Primates in perspective, ed. C. J. Campbell, A. Fuentes, K. C. MacKinnon, M. Panger, S. K. Bearder, 636-651. Oxford: Oxford University Press. 
Symington, M. M. 1988. Food competition and foraging party size in the black spider monkey (Ateles paniscus chamek). Behaviour 105:117-132.

—. 1990. Fission-fusion social organization in Ateles and Pan. International Journal of Primatology 11:47-61.

te Boekhorst, I. J. A., and P. Hogeweg. 1994. Self-structuring in artificial "chimps" offers new hypotheses for male grouping in chimpanzees. Behaviour 130:229-252.

Turnbull, C.M. 1965. Wayward servants: the two worlds of the African pygmies. Westport, Conn: Greenwood.

van Schaik, C. P. 1989. The ecology of social relationships amongst female primates. In Comparative socioecology: the behavioral ecology of humans and other mammals, ed. V. Standen and R. Foley, 195-218. Oxford: Blackwell Scientific Publications.

Ward, P. I., and A. Zahavi. 1973. Importance of certain assemblages of birds as information centers for food finding. Ibis 115:517-534.

Wittemyer, G., I. Douglas-Hamilton, and W. M.Getz. 2005. The socioecolgy of elephants: analysis of the process creating multitiered social structures. Animal Behaviour 69:1357-1371.

Woodburn, J. 1982. Egalitarian societies. Man 17:431-451.

Wrangham, R. W. 1977. Feeding behaviour of chimpanzees in Gombe National Park, Tanzania. In Primate ecology: studies of feeding and ranging behaviour in lemurs, monkeys, and apes, ed. T. H. Clutton-Brock, 504-538. London: Academic Press.

—. 1979. On the evolution of ape social systems. Social Science Information 18:335-368.

—. 1980. An ecological model of female-bonded primate groups. Behaviour 75:262-300.

—. 1999. Evolution of coalitionary killing. Yearbook of Physical Anthropology 42:1-30.

Zahavi, A. 1977. The testing of a bond. Animal Behaviour 25:246-247. 


\section{Figure legends}

Figure 1. A three-dimensional conceptual framework to represent the degree of fission-fusion dynamics of groups and taxa. The $\mathrm{X}$-axis represents the temporal variation in spatial cohesion among group members; the $\mathrm{Y}$-axis represents the temporal variation in party size; and the Z-axis represents the temporal variation in party composition. Region A illustrates cases that are low in all three dimensions such as very cohesive groups or constantly dispersed situations (e.g., territorial, solitary species). Region B would include cases that are highly variable in spatial cohesion and party size, but not in party composition, such as the multilevel societies of hamadryas baboons that are based on the relatively stable one-male units. Region $\mathrm{C}$ represents cases that are high in all three dimensions such as the highly fluid communities of chimpanzees, spider monkeys and spotted hyenas characterized by highly variable party membership. Groups of modern humans, bottlenose dolphins and elephants would likely be located between regions B and C. Comparative data for each dimension are needed for the accurate relative placement of species and populations.

Figure 2. Hypothetical components of a spatially explicit agent-based simulation model. On the left is a photograph of the landscape being simulated. The top layer on the right side of the figure [A] shows "baboon" agents distributed across the landscape at one point in time and showing varying degrees of spatial association (e.g., four "parties” containing 2 to 6 independently-locomoting animals). The bottom two layers on the right represent the spatial distribution of two key resources presumed to be important to baboons at that same point in time, where [B] represents the gradient of food resource availability across the landscape, with the degree of shading proportional to average energy return rate (e.g., calories per unit time) that "baboons” could receive by foraging at that location, and [C] represents the locations of possible sleeping sites (e.g., cliffs) where predation risk is lower (i.e., the degree of shading is inversely proportional to the degree of predation risk experienced at that site). In running a simple simulation, individual "baboons" are given a foraging rule that moves them across the 
landscape (e.g., “move to an adjacent location where the return rate is higher”). At each step, the positions of all "baboons" and the details of each resource layer are updated, and various measures of association (e.g., average inter-individual distance, average party size, etc.) can be calculated.

Figure 3. Alternative routes to higher degree of fission-fusion dynamics. Note that the ecological conditions that require fissioning of cohesive groups along Route A may also make cohesiveness detrimental for higher FF groups along Route B. In this scenario, extended associations may impose ecological costs that would need to be offset by other advantages, such as increased survival of offspring or reproductive success among mates, to achieve the neutrality required for the social transitions along Route B.

Figure 4. Social landscape and its implications for social signals. The X-axis represents the degree of cohesiveness, while Y-axis represents the degree of differentiation of social relationships. The type and frequency of signals required to maintain the corresponding range of social associations emerges as an interaction of the two axes within the social landscape space. The position of species on the landscape is meant to be relative and not absolute. To illustrate the variation that is captured by our framework, several primate species have been placed within the landscape. The utility of the landscape is further demonstrated by the placement of several carnivore species. Polar bears are largely solitary and fall within quadrant I. Dwarf mongooses, wolves and spotted hyenas fall above the X-axis as they are group-living species with highly individualized relationships, but differ in the extent of cohesiveness (Holekamp, Boydson, and Smale 2000). Intraspecific variation may also be illustrated within the landscape. For example, female and male African elephants are positioned along the X-axis to reflect the sex difference in cohesiveness (see electronic supplement B). 
Figure 1

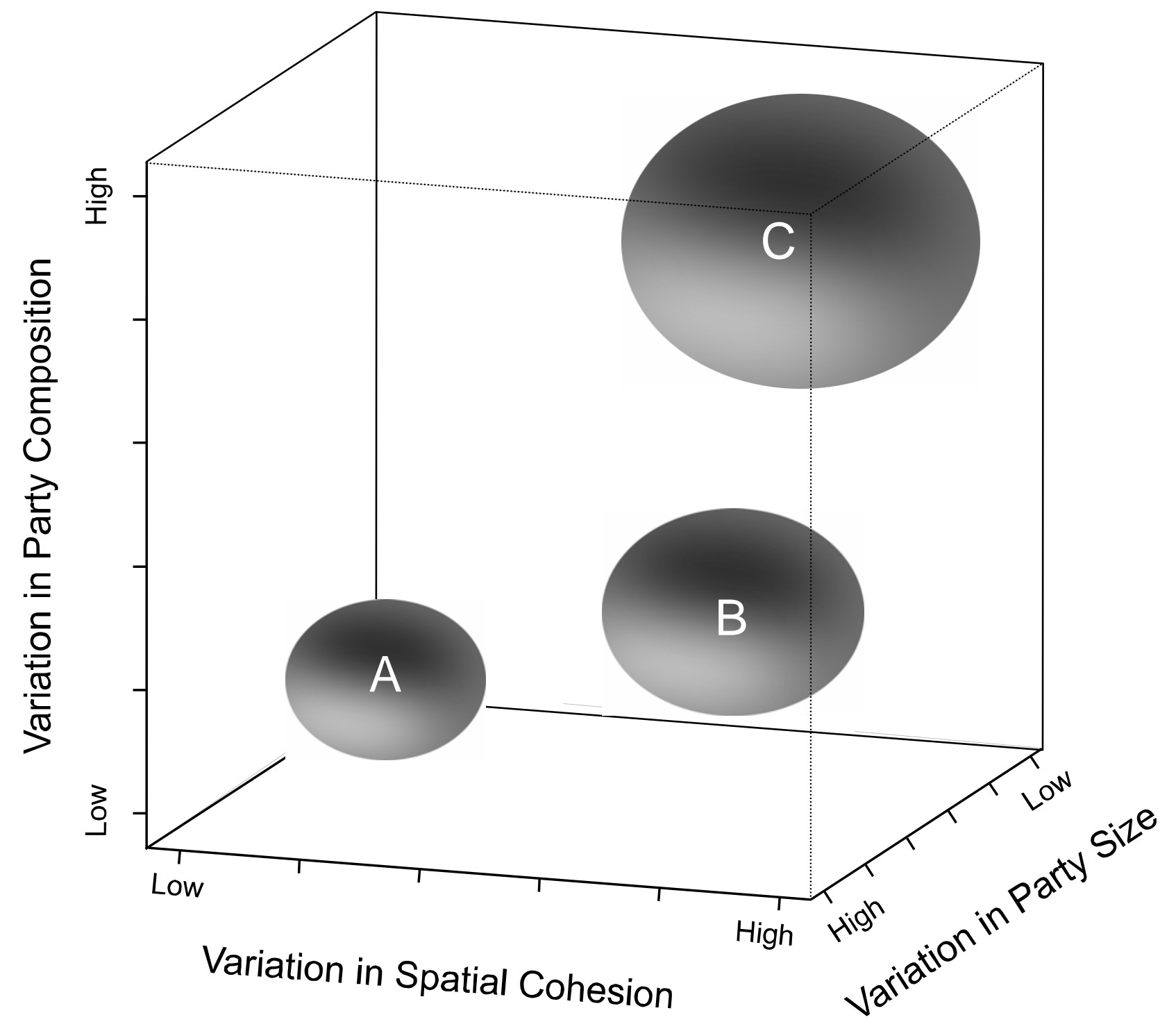


Figure 2

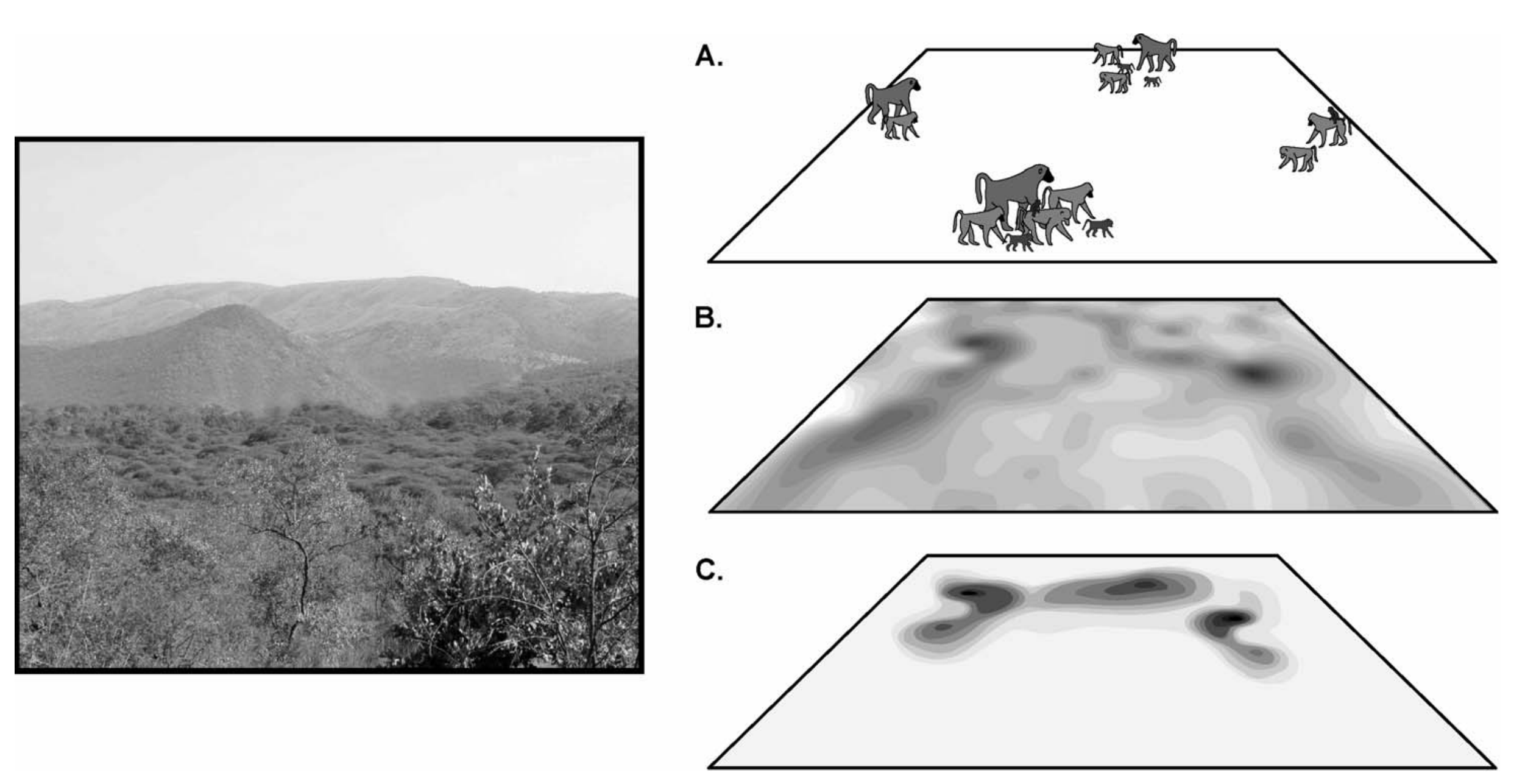


Figure 3

\section{Variable}

Resulting society

Ecological conditions

Spatial associations

Basis and quality of social relationships

Extended associations

(e.g. mates and/or mother-offspring)

Ancestral condition
Route A

Higher FF groups

$\uparrow$

Require fissioning<smiles>CC1CC1</smiles>

Cohesive groups

$\uparrow$

Individual value

Increasingly structured

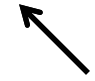

Advantageous
Route B

Facultatively cohesive

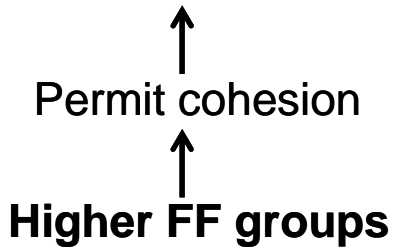

Familiarity

Less structured

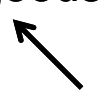

Neutral

Solitary 
Figure 4

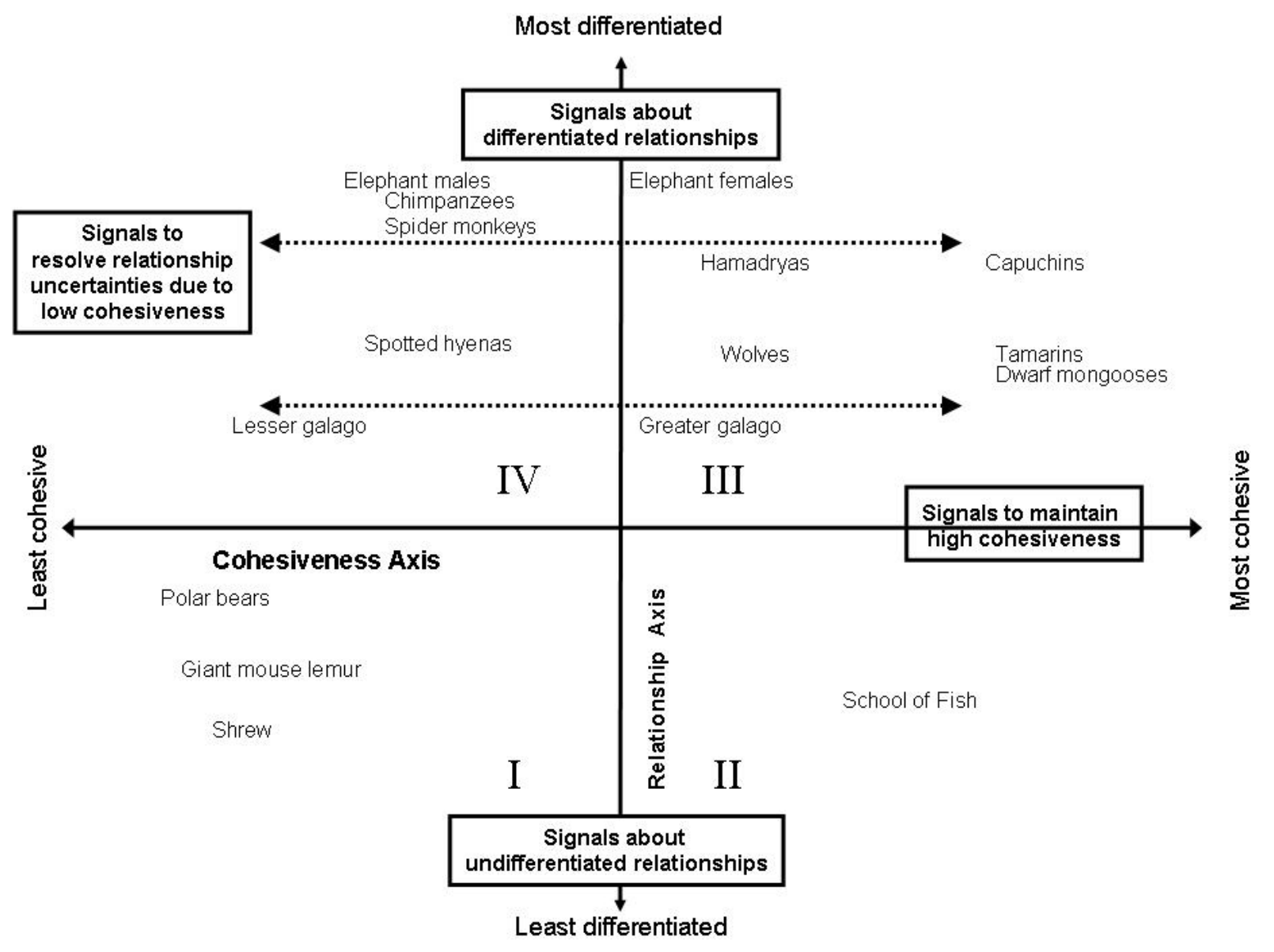

